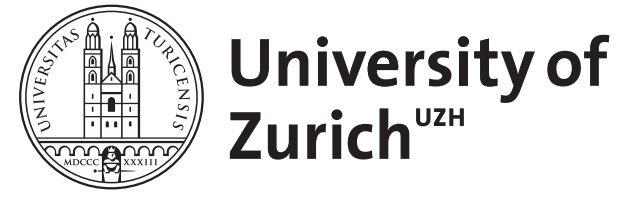

Zurich Open Repository and Archive

University of Zurich

University Library

Strickhofstrasse 39

CH-8057 Zurich

www.zora.uzh.ch

Year: 2015

Welche Fächer wählen Facharztabsolventen?

Djalali, S ; Tandjung, R

Posted at the Zurich Open Repository and Archive, University of Zurich

ZORA URL: https://doi.org/10.5167/uzh-112267

Journal Article

Updated Version

Originally published at:

Djalali, S; Tandjung, R (2015). Welche Fächer wählen Facharztabsolventen? PrimaryCare, 15(14):238239. 


\section{Welche Fächer wählen Facharztabsolventen?}

\section{Sima Djalali, Ryan Tandjung}

Institut für Hausarztmedizin Universität Zürich

Das Weiterbildungsprogramm zum Facharzttitel Allgemeinmedizin wurde seit 1998 mehrfach umgestaltet. Wie sich das ausgewirkt hat, lässt sich aus den Daten der zuständigen Prüfungskommissionen ablesen.

Der Facharzttitel "Allgemeinmedizin» wurde 2011 mit dem Facharzt "Innere Medizin» zusammengelegt zum Facharzt «Allgemeine Innere Medizin». Dies ging mit Änderungen der Weiterbildungsordnung einher. Aber schon in den Jahren zuvor gab es einige Veränderungen. 1998 wurde eingeführt, dass mindestens zwei Jahre Weiterbildung in Ergänzungsfächern geleistet werden können, die die medizinische Breite erweitern,
z.B. in Rheumatologie, Pädiatrie, HNO oder Gynäkologie. 2006 wurde mindestens ein Jahr ambulante Weiterbildung obligat, wobei neben Praxisassistenzen und Poliklinikstellen auch drei Monate pro Jahr in einem stationären Weiterbildungssetting als «ambulante» Medizin angerechnet werden können. Chirurgie, bis dato obligat als Weiterbildungsfach, war nicht länger Pflicht.

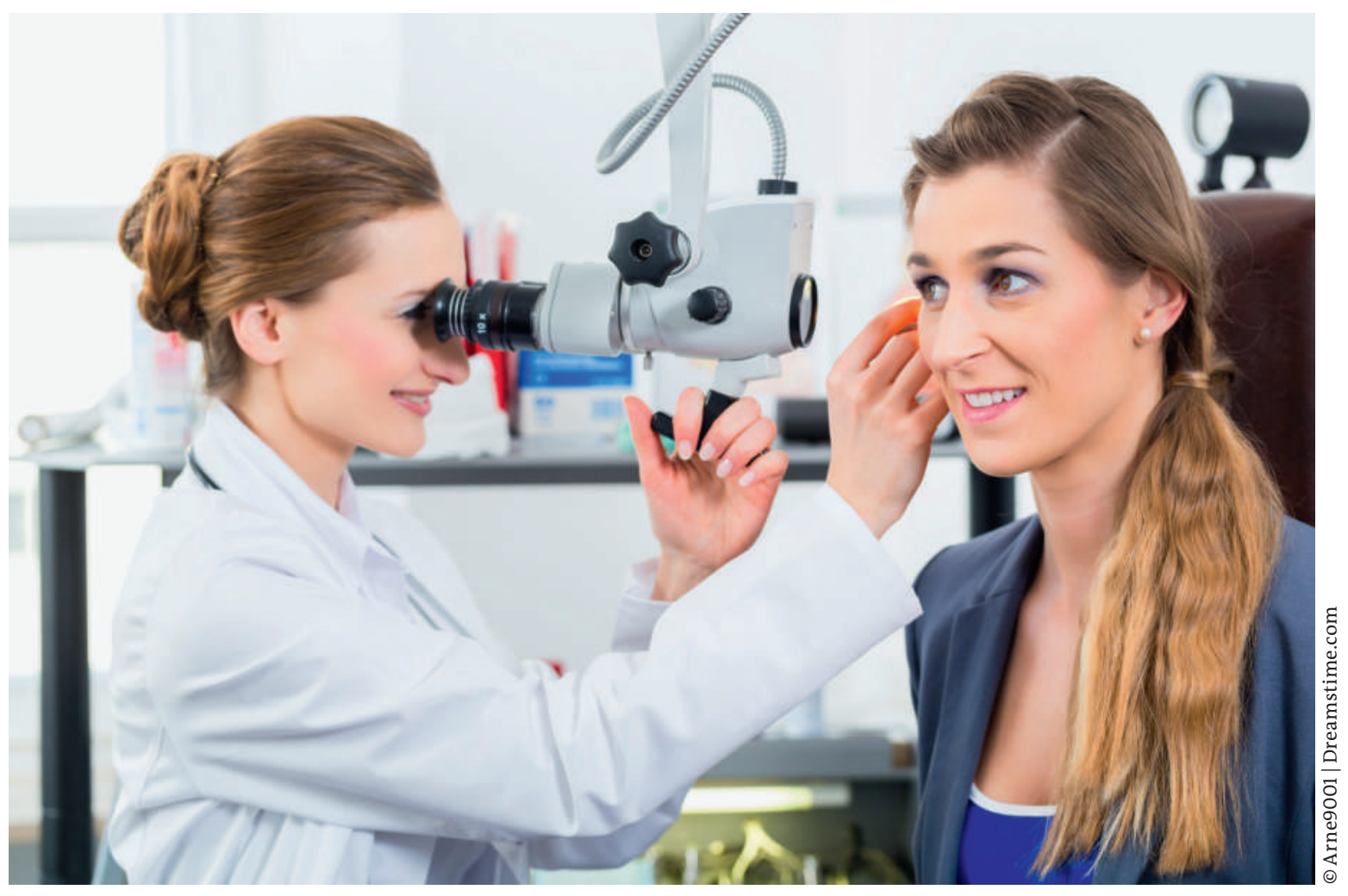

Abbildung 1: Die fachliche Breite der Titelanwärterlnnen nahm über die Jahre ab. 


\section{Keine Pflichtfächer für Hausärzte}

Die regulatorischen Änderungen führten dazu, dass die Fächerzusammenstellungen der Facharztabsolventen sehr heterogen waren. Die Gründung des Titels "Allgemeine Innere Medizin» erlaubt auch weiterhin eine sehr freie Zusammenstellung der Weiterbildungsfächer. Konkret enthält das Programm mindestens zwei Jahre stationäre und sechs Monate ambulante Basisweiterbildung in Innerer Medizin sowie zwei Jahre frei wählbare Aufbauweiterbildung.

Für angehende Hausärzte besteht keine Pflicht, eine spezifische hausarztorientierte Weiterbildung zu absolvieren. Im günstigsten Fall schafft es ein Anwärter, während der Aufbauweiterbildung Weiterbildungsstellen zu finden, die ihm für eine Laufbahn in der Hausarztmedizin gut vorbereiten und fördern.

\section{Prüfungsdaten geben Einblick}

Um mehr darüber zu erfahren, wie die häufigen Änderungen der Weiterbildungsordnung sich auf die Fächerwahl der Absolventen zwischen 1998 und 2012 ausgewirkt haben, wurden die Daten ausgewertet, die die Prüfungskommissionen der Schweizerischen Gesellschaft für Allgemeinmedizin (SGAM) und der FMH über die Jahre gesammelt haben. Dafür wurden 1763 Prüfungen untersucht.

\section{Chirurgie konstant beliebt}

Die Analyse zeigte, dass der jährliche Frauenanteil unter den Titelanwärtern zwischen 1998 und 2007 langsam (um 14\% von 29 auf 43\%) und zwischen 2007 und 2012 stärker (um rund 20 auf 63\%) anstieg. Obwohl Chirurgie nicht länger ein Pflichtfach ist, wurde es auch nach 2006 weiter rege belegt (89,2\% der Titelanwärter 2012).

\section{Fachliche Breite nimmt ab, Praxisassistenz zu}

Die fachliche Breite der Titelanwärter nahm über die Jahre ab. Im Schnitt wurde 2012 ein Ergänzungsfach weniger absolviert als 1998. Dafür haben die Verweilzeiten in Weiterbildungsstellen für Innere Medizin und Allgemeinmedizin zugenommen. Dies ist auch der gestärkten Position der Praxisassistenz zuzuschreiben. Der Anteil von Titelanwärtern, die eine Praxisassistenz absolvierten, verdoppelte sich nahezu in der Beobachtungszeit von 33,3 auf 67,8\%.

\section{Mangel an kurzen Stellen}

Dass die fachliche Breite der Titelanwärter abgenommen hat, könnte daran liegen, dass immer weniger kurzzeitige Stellenangebote in Ergänzungsfächern angeboten werden, da es für Kliniken unattraktiv ist, Assistenzärzte nur kurzfristig zu beschäftigen. Zudem werden Stellen in Spezialfächern bevorzugt an Facharztanwärter aus dem eigenen Fach statt an angehende Allgemeinmediziner vergeben. Dem sollte entgegengewirkt werden, um die fachliche Breite der zukünftigen Allgemeinmediziner und damit der angehenden Hausärzte zu erhalten und zu fördern. Die Organisation und Subventionierung von strukturierten hausarztspezifischen Weiterbildungscurricula sollten verstärkt werden.

\section{Blackbox Hausarztkarriere}

Leider geht aus den untersuchten Datenbeständen nicht hervor, welche Facharztabsolventen schliesslich eine Karriere in der Hausarztmedizin angetreten haben. Um den Einfluss von Förderungsmassnahmen in Zukunft besser einschätzen zu können, sollte die Datensammlung diesbezüglich perfektioniert werden.

\section{Literatur}

Hanhart A, Rosemann T, Marty F, Tandjung R: Weiterbildung zum Facharzt für Allgemeinmedizin in der Schweiz - welche Fächer werden gewählt? Zeitschrift für Allgemeinmedizin. 2014;90(5):234-9.

\section{PrimaryResearch - das Fenster zur Forschung}

In einer Artikelserie stellen wir die Forschungsarbeiten vor, die das Institut für Hausarztmedizin der Universität Zürich $(\mathrm{IH}$ AMZ) seit seiner Gründung publiziert hat. Die Originalarbeiten sind entweder open access zugänglich oder beim jeweiligen Autor, der die Studien hier vorstellen wird, auf Anfrage erhältlich.

Die Ergebnisse geben einen spannenden Einblick in die täglichen Herausforderungen, aber auch die Leistung der Hausarztmedizin.

An dieser Stelle ein herzlicher Dank an alle Kolleginnen und Kollegen, die sich an den jeweiligen Projekten beteiligen und die hier präsentierten Ergebnisse erst ermöglicht haben!

\section{Universität} Zürich ${ }^{\text {vin }}$

Institut für Hausarztmedizin 